\section{NP5 (continued)}

current study aims to examine multiple components of school readiness including behavioral school readiness as well as cognitive school readiness collected from Head Start. In addition, as a post hoc aim we consider associations with early life stress exposure.

Description: Participants $(n=66)$ were preschool children recruited from Head Start for a larger longitudinal study. Behavioral school readiness was assessed using the Children's Behavior Questionnaire-Short Form. Cognitive school readiness was assessed using the Desired Results Developmental Profile (DRDP) obtained from Head Start records. Child height and weight were measured, and used to calculate BMI z-score. Plasma leptin, IL-6, TNF-alpha, and CRP were measured by electro-chemiluminescent immunoassay and turbidimetric assay, respectively. Parents completed questions representing aspects of early life stress, including household income, marital status, and their participation in government assistance programs.

Evaluation: Partial correlations controlling for child age show hypothesized negative associations between proinflammatory cytokines and school readiness, while leptin was positively associated with cognitive school readiness and BMI z-score. Children classified as having the most exposure to early life stressors had significantly lower scores for cognitive school readiness and marginally lower scores for inhibitory control.

Conclusion and Implications: Findings indicate further research on the physiological impact obesity may have in development during early childhood is warranted. In particular, a better understanding of associations between obesity, physiology, and school readiness is needed to provide effective prevention and intervention efforts while these associations are still developing.

Funding: 2015-68001-23280.

\section{NP6 Using Biomarkers for the Validation of Niños Sanos, an Obesity Risk Assessment Tool: Preliminary Results}

Louise Lanoue, PhD, llanoue@ucdavis.edu, University of California, 1 Shields Avenue, Davis, CA 95616;

Marilyn S. Townsend, PhD; Mical K. Shilts, PhD, California State University; Christiana Drake, PhD, University of California, Davis; Lenna Ontai, PhD;

Karina Diaz Rios, PhD, University of California, Merced; Nancy Keim, PhD, USDA Western Human Nutrition Research Center; Dennis M. Styne, MD, University of California, Davis

Objective: Obesity disproportionately affects 2 - to 5 -yearold children from low-income families and among them Latino children have the highest rates. Our goal is to validate a final version of Niños Sanos (NS), a pediatric obesity risk assessment tool designed for use with low-literacy Spanish speaking parents.

Description: This is a cross-sectional study. Parent-child pairs $(\mathrm{n}=167)$ were recruited from Head Start $(\mathrm{n}=19)$ and WIC $(\mathrm{n}=3)$ sites in Yolo and Sacramento counties in CA. Over a 6-week period, parents completed the 45 item NS tool and provided 24-hour dietary, sleep, and activity logs; anthropometrics and blood samples were collected from children. We analyzed (SAS, Version 9.4) a subset of the outcomes listed above.

Evaluation: The parents in our study were 33 year females (>98\%), Hispanics (>99\%), born in Mexico (>83\%), and spoke Spanish primarily ( $>95 \%)$. A majority completed grade school (72\%), most were unemployed (69\%) and lived in a household composed of 2 adults (78\%) and 2 or 3 children (63\%). Thirty percent of the children were overweight and 53\% were obese. A higher NS parental score (healthier behaviors) of the 19 item BMI subscale was associated with smaller child waist circumference $(P=.056)$. A higher parental score on the 3-item NS sweet sugar beverages subscale was associated with a healthier child lipid index (cholesterol, LDLC, Non-HDLC, cholesterol:HDLC, $P=.09$ ), and a lower triglycerides to glucose ratio, an index of insulin resistance $(P=.056)$.

Conclusions and Implications: An obesity risk assessment tool for Spanish speaking families is being validated using biomarkers. The strong association between higher parental NS scores and children with healthier profiles may reach statistical significance with a larger sample size. Niños Sanos promises to be a useful assessment tool to identify the lifestyle determinants specific to Spanish speaking families associated with increased risk of pediatric obesity.

Funding: 2015-68001-23280.

\section{NP7 Advancing and Expanding HomeStyles: Shaping Home Environments and Lifestyles to Prevent Childhood Obesity}

Carol Byrd-Bredbenner, PhD, RDN, FAND,

bredbenner@aesop.rutgers.edu, Rutgers University, 26

Nichol Avenue, New Brunswick, NJ 08901;

Melissa Olfert, RDN, DrPH, West Virginia University;

Karla P. Shelnutt, PhD, RDN, University of Florida

Objective: This multi-disciplinary, multi-institutional, multi-state, integrated Research, Education, and Extension project will advance and expand the progress of HomeStyles toward reducing risk of childhood overweight and obesity.

Description: HomeStyles, an in-home family intervention, enables and motivates English- and Spanish-speaking parents to shape home environments and weight-related lifestyle practices to prevent childhood obesity. Segment 1 of HomeStyles focused on families with preschoolers (ages $2-5$ ). This project will conduct a dissemination feasibility study to determine how to effectively diffuse the innovations of the evidence-based HomeStyles-Segment 1 intervention to parents of children in preschools. This project will create a novel, culturally-competent HomeStylesSegment 2 responsive to the developmentally unique needs of middle childhood (6-11 years) and deliver it online and in face-to-face SNAP-Ed sessions. Further, this project will build the expertise of the next generation of nutrition and health education professionals in creating effective childhood obesity prevention programs through formal coursework and hands-on practicums.

Continued on page $S 110$ 


\section{NP7 (continued)}

Evaluation: The dissemination feasibility study for Segment 1 will include pre/post survey completed by a key informant at each participating preschool/daycare and brief parent surveys. The new, second segment (Segment 2) of HomeStyles tailored to families with children 6 to 11 years will be evaluated via a randomized controlled trial designed to determine whether this novel, age-appropriate, family intervention enables and motivates English- and Spanish-speaking parents to shape their home environments and weight-related lifestyle practices (diet, exercise, sleep) to reduce risk of obesity during middle childhood more than those in the control condition. Students enrolled in coursework and practicums will be evaluated using rubrics and satisfaction surveys.

Conclusions and Implications: This intervention will apply community-based participatory research principles and be aligned with current obesity prevention recommendations and behavior change theories. This project will provide a sustainable model for confronting and combating obesity and safeguarding health that can be brought to scale nationwide.

Funding: 2017-680001-26351.

\section{NP8 Approaches to Enhancing Wellness Policy Implementation in Schools to Prevent Obesity: Implementation}

Erin Hager,PhD, ehager@peds.umaryland.edu, University of Maryland School of Medicine, 737 W Lombard Street, Room 163, Baltimore, MD 21201; Rachel Deitch, MS, CHES; Hannah Lane, PhD, MPH; Amy Zemanick, BS; Katherine Campbell, MPH; Perri Carroll, MPH;

Elizabeth Parker, PhD, RD; Lindsey Turner, PhD, Boise State University; Hee-Jung Song, PhD, University of Maryland College Park; Shauna Henley, PhD, University of Maryland Extension; Brit Saksvig, PhD, University of Maryland College Park

Objective: "Wellness Champions for Change" (WCC) aims to create health-promoting school environments by training teachers and student leaders (5th or 8 th graders) to promote Local Wellness Policy (LWP) implementation. LWPs are school district documents outlining food/ physical activity/health education policies. This abstract describes data collection/intervention implementation for the first cohort of schools/students.

Description: Six schools (3 elementary/3 middle) serving low-income families within one urban school district and 108 students ["evaluation cohort" (EC) followed for 2 years, 4 th-5th and 7 th-8th grade] were recruited. Schools were block randomized to: WCC-teachers + student leaders, WCC-teachers only, control. Measures were based on REAIM (reach, effectiveness, adoption, implementation, maintenance) framework. Effectiveness measures include a new audit tool (OSEC- Observational School Environment Checklist) and EC measures of height/weight (BMIfor-age calculated), physical activity (accelerometry), and diet (PODS- Perceptions of Diet at School screener). Implementation of teacher/student leader curricula is tracked through attendance/activity logs, fidelity checklists, and participant interviews. Adoption/reach will be measured by comparing participating districts/schools to nonparticipants. Maintenance will be assessed over 2.5 years. Evaluation: OSEC measures revealed consistent space available for indoor activity, a lack of menuing and nutrition promotion in cafeterias, limited water access, and lowquality activity resources on school grounds. EC students were $44 \%$ male, average age 11.3 years (range: 9.3-14.2), $39.8 \%$ overweight/obese, and averaged 27.4 minutes/day of moderate-vigorous physical activity. Most lunches were from school cafeteria (48.4\%) or home (31.2\%) and of these, $60.8 \%$ reported consuming a fruit and $44.6 \%$ a vegetable (typical day). In intervention schools, teachers ( $\mathrm{n}=4$ schools) and student leaders ( $\mathrm{n}=2$ schools) are receiving the manualized curricula, which promotes goal-setting, action planning, and implementation. Fidelity checklists and activity logs to track implementation are ongoing.

Conclusions and Implications: The second year of a 5-year project included recruitment of schools/students, baseline data collection, and intervention implementation. New and existing RE-AIM-based measures were employed. Four additional districts (including rural and suburban), up to 36 schools, and nearly 900 students will be recruited and evaluated in future years.

Funding: 2016-68001-24927.

\section{NP9 Building a Cadre of Minority Scientists in the Area of Childhood Obesity Prevention: A Mentoring Initiative}

Mary Murimi,PhD, RDN, mary.murimi@ttu.edu, Texas Tech University, 1301 Akron Avenue, Lubbock, TX 49709-1240; Joseph Sharkey, PhD, MPH, Texas A \& M Health Sciences Center; Tandalayo Kidd, PhD, Kansas State University; Angela Odoms-Young, PhD, University of Illinois, Chicago; Lorraine Weatherspoon, PhD, Michigan State University; Valentine Njike, $M P H, M D$, Yale-Griffin Prevention Research Center

Objective: The objectives of this initiative were: to mentor early career minority investigators to develop their research agenda in the areas of childhood obesity; and to increase collaboration in applied, and translational research in childhood obesity arena.

Description: Despite the fact that there is disproportional prevalence of childhood obesity among minority population compared to their majority counterparts, limited federal funding goes to minority investigators compared to their majority counterparts. In an effort to narrow that disparity gap, developing a mentoring program with mentors that have a clear understanding of the obstacles faced by minority scholars is promising. Mentoring offers an opportunity for knowledge exchange and integration, with the mentee investigators sharing their novel ideas and culture, while the mentor provides the technical knowledge, experience, and implementation of a grant project. Mentoring results in a learning partnership that enhances professional growth and development. The

Continued on page S111 